\title{
Gesundheitsbegriffe in der Psychiatrie
}

\begin{abstract}
Concepts of Health in Psychiatry. In talking about concepts of health in psychiatry, we are not talking about an essentialist concept of health, where there is some essential thing that health might be. There is a straightforward sense in which psychiatric „health" simply means the absence of psychiatric disease. Marking out our concepts of health in psychiatry would then involve marking out the boundaries between normal and abnormal psychic phenomena. However, there is no single, neat concept of health in psychiatry; nor are there concepts of health that neatly cohere into one overarching theory of health. This is not because psychiatry is vague. It is because psychiatry reflects the complexity of the whole person.
\end{abstract}

\section{Einführung}

Wenn wir über Gesundheitsbegriffe in der Psychiatrie sprechen, geht es uns gewiss nicht um einen essentialistischen Begriff, als ob die Gesundheit ein Gegenstand mit bestimmten notwendigen Eigenschaften wäre. Oder um es anders auszudrücken: Es soll nicht behauptet werden, Begriffe würden konkrete Dinge bezeichnen. Vielmehr wollen wir in post-Wittgenstein'scher Manier davon ausgehen, dass wir die Bedeutung des Wortes ,Gesundheit" dann verstanden haben, wenn wir verstanden haben, wie es gebraucht wird, d.h., wie es im konkreten Leben funktioniert. Wenn das Wort, Gesundheit ' im Alltag vielfältige Bedeutungen annehmen kann, liegt es also nahe, dass es auch im psychiatrischen Kontext verschiedene Bedeutungen besitzen wird. Natürlich bedeutet ,Gesundheit in der

\footnotetext{
Anmerkung: Der ausgesprochene Dank des Autors gilt Dorothea Keller für die Übersetzung des vorliegenden Kapitels aus dem Englischen und Professor Philip van der Eijk für die Durchsicht der Übersetzung sowie Professor Hanfried Helmchen, der das Kapitel freundlicherweise gelesen und einige hilfreiche Anmerkungen beigesteuert hat, die zweifellos geholfen haben, einige Passagen und Ausdrücke deutlicher zu gestalten. Ferner dankt der Autor Herrn Dr. Roman Marek, der den Text durch Beisteuern weiterer Vorschläge bereichert hat. An dieser Stelle seien außerdem noch Hinweise auf weiterführende Literatur deutscher Sprache gegeben, die ebenfalls auf Prof. Helmchen zurückgehen. Sie können als Kommentare zu den Gedanken des Autors verstanden werden: Fuchs 2010; Heinz 2014.
}

Julian C. Hughes, Bristol Medical School: Bristol Population Health Science Institute, University of Bristol

Ә OpenAccess. (c) 2021 Julian C. Hughes, publiziert von De Gruyter. (cc))BY-NC-SA Dieses Werk ist lizenziert unter einer Creative Commons Namensnennung - Nicht kommerziell - Weitergabe unter gleichen Bedingungen 4.0 International Lizenz. https://doi.org/10.1515/9783110713336-022 
Psychiatrie zunächst einmal schlicht die Abwesenheit psychischer Erkrankungen. Dementsprechend bestünde die Bestimmung von Gesundheitsbegriffen in der Psychiatrie darin, Grenzlinien zwischen normalen und abnormen psychischen Phänomenen zu ziehen.

\section{Die Eingrenzung des Normalen}

Hierzu sind zunächst zwei Punkte anzumerken. Erstens ist eine solche Grenzziehung natürlich schon unternommen worden. Das ganze Arbeitsgebiet, das man als Psychopathologie bezeichnet, beschäftigt sich konkret damit, gewissermaßen normale und abnorme psychische Erfahrungen voneinander abzugrenzen. Karl Jaspers beispielsweise erreicht dieses Ziel in seiner grundlegenden Arbeit von 1913, in der er eine philosophische Methode vorlegt, um abnorme psychische Phänomene darzustellen. Das Abnorme dient zur Beschreibung des Normalen. Mit anderen Worten: Wenn wir exakt definieren, was es bedeutet, an Wahnvorstellungen zu leiden, dann lässt sich Gesundheit offensichtlich nicht mit Überzeugungen vereinbaren, die jemanden glauben machen, er sei für alles Übel in der Welt verantwortlich, bloß weil er eventuell etwas unhöflich zu der Frau beim Bäcker war. Stellt man sich auf der anderen Seite jemanden vor, den seine Angstzustände nicht daran hindern, souverän vor Vorgesetzten aufzutreten, so ist gleichermaßen klar, dass er in dieser Hinsicht als gesund anzusehen ist. Wenn eine Person aber wegen ihrer Angstzustände unter allen Umständen vermeiden will, überhaupt zur Arbeit zu gehen, dann liegt schon eher eine pathologische Situation vor.

Wir sollten diese beiden Beispiele dennoch nicht vorzeitig als offensichtliche Tatsachen akzeptieren. Es gibt ja Leute, die Vorstellungen haben, welche den Kriterien von Wahnvorstellungen entsprechen, in ihrem Leben aber sehr gut zurechtkommen und sich durch ihre scheinbar abnormen Überzeugungen nicht allzu sehr beeinträchtigen lassen. Auf der anderen Seite mag Ängstlichkeit kontrollierbar genug sein, um im Berufsleben souverän auftreten zu können, und kann sich dennoch spürbar auf Kosten anderer Lebensbereiche auswirken, etwa durch anderweitige Phasen der Niedergeschlagenheit, in denen der Betroffene unter seinen Möglichkeiten bleibt, durch stundenlanges Proben, Gereiztheit dem familiären Umfeld gegenüber usw. Eine trotz Wahnvorstellungen zufriedene Person erscheint dann gesünder als jemand, der unter ,normaler “ Ängstlichkeit leidet. Das führt uns zum zweiten Punkt, der sich um Wertvorstellungen dreht. 


\section{Wertvorstellungen}

Wenn wir die Grenze zwischen dem psychisch Normalen und Abnormen untersuchen, stoßen wir auf einen Aspekt, der bereits vielfach Forschungs- und Untersuchungsgegenstand geworden ist, nämlich auf die Rolle, die Werturteile in der Psychiatrie spielen. Der Ansatz des wertorientierten Handelns (values-based practice), wie Bill Fulford ihn aus der allgemeinen Sprachphilosophie hergeleitet hat, zeigt zum einen, dass Wertvorstellungen in der Medizin und insbesondere in der Psychiatrie allgegenwärtig sind, und zum anderen, dass Werturteile daher unumgänglich sind - auch dann, wenn es an die Diagnose geht (Fulford et al. 2012; Fulford \& van Staden 2013). Hier zeigt sich, dass wertorientiertes Handeln sogar in der organischen Psychiatrie ${ }^{1}$ fruchtbar angewandt werden kann, einer Disziplin, in der doch konkrete Fakten (beispielsweise über Demenz) zur Verfügung stehen, durch die, so könnte man meinen, die Diskussion von Werturteilen obsolet werden sollte (Hughes \& Williamson 2019). Es gäbe viel über Werturteile und die Abgrenzung zwischen normalen und abnormen psychischen Phänomenen zu sagen - an dieser Stelle soll jedoch der Hinweis darauf genügen, dass das jeweilige Verständnis von Gesundheit immer wertgeladen ist, insbesondere wenn Grenzfälle verhandelt werden.

Um ein konkretes Beispiel zu nennen: Es wäre interessant zu überlegen, wie aufgedreht jemand sein kann, ehe man ihn als ,manisch“ bezeichnen würde. Gegebenenfalls wird ein hypomanischer Zustand, der sich in Kreativität und Tatendrang ausdrückt, sogar positiv erlebt. Kann man das gesund nennen? Der Betroffene mag behaupten, er fühle sich kerngesund, und doch beginnen andere, ernsthaft an seiner geistigen Gesundheit zu zweifeln. Man holt eine Beurteilung von dritter Seite ein, um herauszufinden, wer recht hat. Urteile, die die geistige Gesundheit betreffen, sind also wertend, und zwar wohlgemerkt in höherem Maße als solche, die sich auf die physische Gesundheit beziehen, obgleich (zumal in Grenzfällen) Werturteile auch dort im Spiel sind. Wir könnten etwa fragen, ab wann aus pfeifendem Atem Asthma wird oder ab welchem Wert wir von erhöhtem Blutdruck sprechen wollen.

\footnotetext{
1 ,Organische' Psychiatrie ist das Gebiet, das sich fest auf die Pathologie des Gehirns stützt und damit im Gegensatz zur ,funktionalen` Psychiatrie steht, in der die organische Grundlage der Störung nicht berücksichtigt wird, wie z. B. im Falle von Schizophrenie. Diese alte Trennung zwischen ,organischen` und ,funktionalen` Krankheiten ist jedoch stückweise aufgegeben worden, da die Hirnforschung mittlerweile ein besseres Verständnis der organischen Grundlagen etlicher Krankheiten ermöglicht, die vormals als ,funktional' eingestuft worden waren, etwa Depression oder auch Schizophrenie.
} 
Natürlich gibt es Kriterien, um diese und ähnliche Fragen zu beantworten. Aber solche Kriterien beruhen ihrerseits auf einem Urteil darüber, wie groß die Abweichung vom jeweiligen Normwert ausfallen darf, die einem gesunden Menschen zuzumuten ist. Wir wollen diese Probleme beiseitelassen und lediglich eines festhalten: Dass Urteile über geistige Gesundheit in höherem Maße wertend sind, hat seine Ursache gerade in der Natur des Geistigen. Der Geist ist der Punkt, der es der Psychiatrie so schwer macht zu entscheiden, was gesund und normal ist und was nicht.

\section{Begriffe und Modelle}

Was verstehen wir unter dem Geist? - In der Philosophie ist diese Frage wirklich ein alter Hut und kann hier nicht ausführlich beantwortet werden (Matthews 2005). Ich möchte lediglich hervorheben, dass unser Verständnis des Geistes unsere Begriffe des Geistes - oft auf modellhaften Vorstellungen beruhen. Die Begriffe klinken sich sozusagen in die Modelle ein.

Ich will versuchen, diese Idee anhand dreier Modelle des Geistes noch ein Stück weiter zu verfolgen. Im Fokus stehen ein biologisches oder materialistisches Modell, ein funktionalistisches Modell, das sich den Geist wie einen Computer oder Prozessor vorstellt, sowie ein sozialkonstruktivistisches Modell (mehr zu diesen Modellen und der Natur des Geistes im Zusammenhang mit Demenz in Hughes 2016). Dabei handelt es sich angesichts der Vielzahl von Modellen, die in der Psychiatrie zur Anwendung kommen (Tyrer \& Steinberg 2005), natürlich um eine vereinfachende Auswahl; später unternehme ich einen kurzen Exkurs in die psychodynamische Theorie.

\subsection{Ein biologisches Modell}

Ich fasse diesen ersten Modelltypus als physikalistisch oder materialistisch auf; man könnte auch von einem biomedizinischen oder einem Krankheitsmodell sprechen. Es geht davon aus, der Geist sei nichts weiter als das Gehirn. Wenn ich also psychische Probleme habe, stehen die Ursachen dafür - nach meiner grob verkürzten Darstellung des Modells - in irgendeinem Zusammenhang mit dem Gehirn. Es handelt sich um eine grobe Verkürzung, weil es sich so anhört, als könnten psychische Störungen demnach ausschließlich medikamentös oder invasiv behandelt werden. Aber das Modell schließt psychologische oder soziale Hilfe natürlich nicht aus, da auch psychosoziale Ansätze hinsichtlich ihrer Wirkungen auf das Gehirn betrachtet werden können. Körperliche Ertüchtigung sti- 
muliert beispielsweise die Freisetzung von Endorphinen und steigert so das Wohlbefinden: Die Wirksamkeit körperlicher Ertüchtigung gegen Depression wird durch gutestatistische Befunde gestützt (Schuch et al. 2016).

Was besagt dieses Modell also für unsere geistige Gesundheit? Es legt nahe, dass gesund bleibt, wer sein Gehirn gesund hält.

Personen, die Probleme mit ihrer psychischen Gesundheit haben, bekommen oft zu hören, im Gehirn bestehe ein chemisches Ungleichgewicht: Serotoninmangel im Falle von Depressionen, Dopaminüberschuss bei Psychose, Acetylcholinmangel bei Demenz usw. Die Realität gestaltet sich jedoch deutlich komplexer, als dass sie sich einfach durch Korrelationen zwischen bestimmten Neurotransmittern und der Symptomatologie erklären ließe. Tatsächlich scheint die Annahme, Depression werde durch Serotoninmangel verursacht, sogar falsch zu sein, auch wenn Serotonin unser Gefühlsleben nachweislich beeinflusst (Cowen \& Browning 2015).

In einer Hinsicht spricht allerdings einiges für dieses Krankheits- oder biologische Modell des Geistes. In Bezug auf Demenz wird ja oft betont, dass dem Gehirn guttut, was auch gut für das Herz ist. Eine einflussreiche Untersuchung zur Demenzprävention kommt, statistisch bestens fundiert, zum Schluss, dass Behandlung von Bluthochdruck, Beschränkung im Rauchen, Kontrolle über Diabetes und Übergewicht - alles Dinge, die ebenso förderlich in Bezug auf das HerzKreislauf-System sind -, außerdem höhere Bildungsstandards im Kindesalter sowie Prävention von Gehörverlust und Depression Maßnahmen sind, die ein Drittel der Demenzfälle verzögern oder ganz verhindern könnten (Livingston et al. 2017). Eine aktuelle Studie, die von Almeida-Meza, Steptoe und Cadar (AlmeidaMeza et al. 2020) vorgelegt wurde, betont die Bedeutung psychosozialer Faktoren für die Demenzprävention: Die Untersuchung legt nahe, dass höhere Bildungsstandards, komplexe Tätigkeiten in der Lebensmitte und eine Kombination aus geistig anregender und sportlicher Freizeitgestaltung im Alter die sogenannte kognitive Reserve erhöhen und dadurch vor Demenz schützen könnten. Dem liegt die Annahme zugrunde, dass (vereinfachend gesprochen) kognitive Reservekapazitäten dazu beitragen, dass das Gehirn den zerstörerischen Alterungsprozessen besser widersteht und so eine Demenzerkrankung vermeidet: Dieser Punkt unterstreicht einmal mehr die Bedeutung des Gehirns für alle psychischen Belange. Also: Bleibende psychische Gesundheit beruht darauf, das Gehirn gesund zu erhalten.

Es überrascht wenig, dass Schädeltraumata auch psychiatrisch betrachtet nicht gut für die Gesundheit sind. Aber reicht das biologische Modell aus, um psychische Gesundheit zufriedenstellend zu beschreiben? Es gibt einige Gründe, die gegen dieses Modell sprechen - ich nenne nur drei: 
Erstens gibt es keine strenge Korrelation zwischen Hirnverletzungen und dem Grad der geistigen Einschränkung. Ich führe noch ein anderes Beispiel aus der Forschungsliteratur zur Demenz an: In einer Reihe von Veröffentlichungen im Rahmen der berühmten „Nonnenstudie“, die eine Gruppe amerikanischer Ordensschwestern bis zu ihrem Tod begleitet und danach Gewebeproben untersucht hat, ließ sich zeigen, dass Pathologie und Symptomatik sich einander nicht eindeutig zuordnen lassen: Obwohl sie neuropathologische Läsionen aufwiesen, die sich in Bezug auf Lokalisierung, Typ und Ausmaß annähernd glichen, zeigten die Teilnehmerinnen der Studie eine erstaunliche Bandbreite an klinischen Erscheinungsbildern, von völliger Symptomfreiheit bis hin zu schwerwiegender Symptomatik (Snowdon 2003, S. 453). Ganz sicher ist einem Gehirn nicht einfach anzusehen, ob zu Lebzeiten bestimmte Anzeichen schlechter geistiger Gesundheit anzutreffen waren.

Wenn wir zweitens an bestimmte intellektuelle Einschränkungen, zum Beispiel an die mit Trisomie 21 verbundenen, denken, müssen wir annehmen, dass sich das Gehirn der betroffenen Person in einer gewissen Hinsicht abnorm verhält: Dennoch sollten wir uns davor hüten, diese Person als nicht gesund zu bezeichnen. Natürlich können anderweitige physische Erscheinungen auftreten, die oft in Verbindung mit bestimmten Syndromen stehen (beispielsweise weisen Kinder mit Down-Syndrom überdurchschnittlich oft Herzfehler oder eine Ösophagusatresie auf, eine Fehlbildung, bei der die Speiseröhre - der Ösophagus - nicht bis zum Magen reicht), aber viele Menschen, die mit der Diagnose Down-Syndrom leben, erscheinen sonst kerngesund und glücklich. Dieser Punkt führt uns zu zwei weiteren Erwägungen.

Erstens liegt eine Unterscheidung zwischen subjektiver und objektiver Perspektive nahe; es existiert eine große Bandbreite an Auffassungen des Begriffs ,Wohlbefinden' (Griffin 1986). Manche rein objektiv orientierte Einschätzung dagegen verleitet dazu, eine Person mit geistiger Behinderung als nicht gesund zu bezeichnen (auch wenn viele dagegen Einspruch erheben würden, und das mit gutem Grund). Wer aber wollte dieser Person absprechen, dass sie sich subjektiv gesund und munter fühlt? Entscheidend ist, dass die geistige Behinderung nicht in Zusammenhang mit einem Gesundheitszustand steht, der sich chronisch verschlechtert, so dass das Bekenntnis des Betroffenen, sich gerade geistig fit und zufrieden zu fühlen, zutreffend erscheint. Der springende Punkt ist, dass wir solche Unterschiede werten.

Außerdem kann der Begriff der ,Behinderung، auf zwei unterschiedliche Weisen verstanden werden: Eine Person ist behindert durch eine körperliche Einschränkung, die ihren Aktionsradius begrenzt; sie macht aber auch die Erfahrung, durch unangemessene soziale Reaktionen anderer stigmatisiert und so zusätzlich behindert zu werden. Problematischerweise legt das biologische bzw. 
Krankheitsmodell nahe, von einer besonderen Gestalt des Genotyps darauf zu schließen, dass der Phänotyp nicht gesund sein könne - und genau solche Urteile sind ausgesprochen fragwürdig.

Daraus folgt der dritte Einwand gegen das biologische Modell: Es gilt, zwischen einer kausal und einer konstitutiv orientierten Betrachtungsweise des Geistes zu unterscheiden. Der kausale Zugang beschreibt die biologischen Ursachen für eine bestimmte Geistesregung. Er lässt sich beispielsweise leicht auf Schizophrenie anwenden: Sobald wir uns auf eine Form der Schizophrenie festgelegt haben (was, wohlgemerkt, Werturteile impliziert), gibt es gute Gründe dafür, manche der Symptome über einen hirnpathologischen Ansatz zu erklären. Eine aktuelle Studie (Osimo et al. 2019) beschäftigt sich beispielsweise mit Synapsenschwund. Die Synapse ist der Ort, an dem Nervenzellen aufeinandertreffen und über chemische Neurotransmitter Informationen austauschen. Das Protein Synaptophysin gilt dabei als guter Gradmesser für die Dichte der interneuronalen Synapsen. Osino und sein Team konnten mit ihrer Untersuchung eine mäßige bis starke Verminderung von Synaptophysin in zwei Gehirnregionen, Hippocampus und Frontallappen, nachweisen. Wie derartige Ergebnisse zu interpretieren sind, bleibt im Letzten bislang unklar. Dennoch gibt es Hinweise auf einen möglicherweise zwingenden Kausalzusammenhang zwischen Gehirnbiologie und Schizophrenie.

Konstitutiv orientierte Zugänge auf der anderen Seite, die sich im weiteren Sinne der Phänomenologie zuordnen lassen, beschreiben, wie es sich tatsächlich anfühlt, diese oder jene Geistesregung zu erleben. Wie geht es jemandem wirklich, der an Schizophrenie leidet? An dieser Stelle wird der Phänomenologie psychischer Erfahrungen nachgegangen, nicht ihren physischen Ursachen. Beide Ansätze, der kausal und der konstitutiv orientierte, liefern uns nützliche Informationen über Geisteszustände. Dennoch gibt es einiges, was der konstitutive Zugang dem kausalen voraus hat (was selbstverständlich auch umgekehrt gilt). Jemand, der ein Verständnis davon hat, wie es sich tatsächlich anfühlt, an Halluzinationen oder Wahnvorstellungen zu leiden, der sich die Angst vorstellen kann, die solche Erfahrungen mitunter auslösen, der bringt ein größeres Einfühlungsvermögen mit, so dass sich eine andere Person, die solche Erfahrungen durchlebt, von ihm eher verstanden fühlen wird. Jaspers Unterscheidung zwischen Erklären und Verstehen ist hier ganz am Platze. Der konstitutive Zugang zielt letztlich darauf ab, zu verstehen, was bestimmte Erfahrungen bedeuten.

Das biologische bzw. Krankheitsmodell dagegen gibt uns überhaupt keinen solchen konstitutiven Zugang. Es kann ihn uns auch gar nicht geben; er will an anderer Stelle gesucht werden. Das biologische Begriffsmodell kann uns also nicht viel darüber sagen, was Gesundheit in der psychiatrischen Praxis bedeutet. Es beschreibt die physische Grundlage guter geistiger Gesundheit - dass etwa 
unsere Gene eine Rolle spielen, dass wir Schädeltraumata vermeiden und auf unsere physische Gesundheit Acht geben sollten, um das Gehirn gesund zu halten usw. - aber es sagt uns nicht, worin gute psychische Gesundheit besteht.

\subsection{Ein Computer-Modell}

Mit gutem Grund wird das Gehirn oft mit einem Computer verglichen. Die kognitive Neuropsychologie vermittelt nützliche Erkenntnisse über die Hirnfunktionen, indem sie einzelne und relativ präzise eingegrenzte neuronale Läsionen und die daraus entstehenden Störungen betrachtet. Auf diese Weise lassen sich Flussdiagramme generieren, die zeigen, wie das Gehirn Informationen verarbeitet. Man kann z. B. ein Wort anhand seines Lautbildes (der Phoneme) oder anhand seines Schriftbildes (der Grapheme) analysieren; alternativ kann man das Wort als Einheit im auditiven Input-Lexikon wahrnehmen. Die anschließende semantische Verarbeitung befähigt zu einem Output, etwa in Form eines geschriebenen Wortes über das graphemische (oder orthographische) Output-Lexikon. Dieses Modell mag sehr raffiniert erscheinen, im Grunde geht es aber nur von Input, Analyse und Output aus. Die Hirnregionen, in denen die Analyse stattfindet, stellt man sich als Lexika vor, in denen Laut- oder Schriftbild mit dem internen Bestand an Laut- und Schriftbildern abgeglichen werden. Indem es die erforderliche Vorstellung an ein Output-Zentrum übermittelt, kann es in mündlicher oder schriftlicher Form den jeweils passenden Output erzeugen. Anhand dieses Modells lässt sich gut rekonstruieren, wie sich ein lokal begrenzter Schlaganfall auswirkt: Der Betroffene kompensiert das Ablaufproblem im Gehirn, indem er einen anderen Weg für die Informationsvermittlung sucht.

Dennoch beinhaltet das Modell philosophische Anleihen: Am augenfälligsten ist die Nähe zum Funktionalismus. Funktionalisten betrachten geistige Zustände als Kausalfaktoren, die das Verhalten eines Subjektes in der jeweiligen Situation mitbestimmen (Lowe 2000, S. 45). Der wohl bekannteste Funktionalist war Jerry Fodor (1935-2017). Er vertrat die Ansicht, dass geistige Regungen (Motive, Emotionen, Intentionen usw.) kausal miteinander zusammenhängen, dass sie gemeinsam eine systematische Sprache bilden, und dass diese Sprache des Geistes aus geistigen Repräsentationen besteht, die dazu dienen, unser Verhalten zu erklären (z. B. Fodor 1976).

Was kann uns dieses Konzept über geistige Gesundheit sagen? Gewissermaßen handelt es sich nur um eine Verfeinerung des biologischen Modells: Während das biologische Modell sich daran orientiert, was physiologisch passiert, geht das Computermodell einen Schritt weiter und betrachtet, was auf funktionaler Ebene geschieht. Wenn ich einen kleinen Schlaganfall in einem klar eingegrenzten Be- 
reich meines Gehirns erleide, dann bedeutet das vielleicht für die pathophysiologische Ebene, dass neuronale Funktionen ausfallen - für die funktionale Ebene bedeutet es, dass ich geschriebene Wörter zwar lesen und verstehen, aber nicht aussprechen kann (auch wenn ich sie vielleicht sogar noch schreiben kann). Das Computermodell erweist sich also als durchaus nützlich und bleibt doch anfällig für dieselben Kritikpunkte wie das biologische Modell.

Der damit implizierte Begriff betrachtet Gesundheit als gegeben, solange die Verbindungen des inneren Computers intakt und die Informationsverarbeitung gewährleistet sind. Darüber hinaus stützt es einige der oben genannten Annahmen, etwa, dass Bildung und soziale Stimulation gut für unsere psychische Gesundheit sind, weil sie die Datenmenge erhöhen, die das Gehirn als computerähnlicher Mechanismus verarbeitet. Wir können uns vorstellen, wie immer neue Verbindungen entstehen und das Gehirn zur optimalen Funktionstüchtigkeit und zu maximalen kognitiven Reserven gelangt. Wo also liegt das Problem an diesem Modell des Geistes?

Einmal mehr kommen wir auf die bereits angestellten Beobachtungen zurück: Während das Computer-Modell Dinge gut kausal erklären kann, erklärt es dennoch nicht, was nun genau diesen oder jenen Geisteszustand ausmacht. Es sagt uns nicht, was es heißt, aus psychologischer Perspektive gesund zu sein. Es erläutert lediglich, inwiefern die Verarbeitung von Geistesregungen sich mit Computerprozessen vergleichen lässt. Doch das Modell birgt noch ein weiteres Problem, und zwar auf einer eher philosophischen Ebene.

Zumindest insofern es von Fodors Funktionalismus beeinflusst ist, hängt das ganze Modell von geistigen - inneren - Repräsentationen $\mathrm{ab}^{2}{ }^{2}$ Die Schwierigkeit besteht nun darin, dass solche internen Repräsentationen in keinem oder in nur sehr geringem Zusammenhang mit der Außenwelt stehen müssen. Sie sind auf einer subpersonalen Ebene anzusiedeln. Natürlich erhält das System ganz am Anfang einen Input, aber dieser braucht nur so beschaffen zu sein wie der Input, den eine Maschine erhält: Er muss nicht notwendig etwas bedeuten. Das Gehirn handelt (nach dieser Sichtweise) nicht anders als ein Computer, der imstande ist, einen Text laut vorzulesen. Der entscheidende Unterschied liegt darin, dass der Text für die Person eine Bedeutung haben sollte; für den Computer braucht er weder bedeutungsvoll $\mathrm{zu}$ sein noch ist er es denn wirklich. In einer gewissen Weise liest und verarbeitet das Gehirn den Text durchaus wie ein Computer, aber es tut eben weit mehr als nur das (solange keine Störungen auftreten), denn es

2 Unter der Bezeichnung ,Repräsentation` sei hier eine Art Informationseinheit verstanden, mit der der Geist operiert. Sie lässt sich näherungsweise beschreiben als das ,innere Bild“ einer Sache. Es gibt neben den bildlich-anschaulichen aber auch symbolische Repräsentationen, auf die der Geist zurückgreift, wenn er ein nicht-anschauliches, abstraktes Konzept vergegenwärtigt. 
erfasst auch den Sinn des Textes. Subpersonale (innere) Repräsentationen jedoch haben ohne jegliche Einbettung in den Kontext der (äußeren) Umwelt der Person keinen Sinn (Hughes 2011, S. 131-145). Der Organismus versteht also etwas nur, weil er das Gehirn einer Person ist. Er ist unmittelbar im Kontext der menschlichen Welt situiert, in dem Dingen eine Bedeutung zukommt.

Was hier nur angedeutet ist, bahnt uns den Weg zu dem, was tatsächlich einen sinnvollen Gesundheitsbegriff in der Psychiatrie ausmachen kann. Wir beginnen, den Geist als mehr anzusehen als bloß das Gehirn, als etwas, das im Kontext von Personen situiert ist. Ein gesunder Geist ist folglich einer, der im weiteren Umfeld der menschlichen Welt den richtigen Platz einnimmt. Das führt uns zur Betrachtung der sozialen Welt, jedoch nicht bevor wir einen sehr kurzen Exkurs in die psychodynamische Theorie unternommen haben.

\subsection{Psychodynamische Theorie}

Ich habe das Computer-Modell als Beispiel für ein psychologisches Modell angeführt. Dabei sei an dieser Stelle der Diskussion noch ein kleiner Vorbehalt ausgesprochen: Es mag andere psychologische Modelle geben, auf die Kritik, wie ich sie am Computer-Modell geäußert habe, nicht zutrifft. Ein Beispiel dafür ist das psychodynamische Modell des Geistes. Psychodynamische Theorie (nicht, dass es nur die eine gäbe!) macht einige Aussagen über Bedeutung und Kontext, den Kontext der individuellen Entwicklung und den Umgang mit Lebensereignissen mit eingeschlossen. Als Modell setzt sie verschiedene Aspekte des Geistes voraus, hauptsächlich die Unterscheidung zwischen Unbewusstem und Bewusstem und die Annahme, Ersteres beeinflusse das Letztere. Man kann das auf eine mechanistische Weise verstehen, wie die frühen Schriften Freuds zeigen. Aber der entscheidende Aspekt der psychodynamischen Psychotherapie liegt darin, dass subpersonale, unbewusste Prozesse mit der bewussten Welt verbunden werden, in der sie Sinn und Bedeutung erhalten.

So ist es möglich, aus der psychodynamischen Theorie eine Art Gesundheitsbegriff herzuleiten. Wenn Freud und Breuer am Ende der Studien über Hysterie (1895) erklären,

dass viel damit gewonnen ist, wenn es uns gelingt, Ihr hysterisches Elend in gemeines Unglück zu verwandeln. Gegen das letztere werden Sie sich mit einem wieder genesenen Nervensystem besser zur Wehre setzen können (Freud \& Breuer 1895, S. 269),

wird „gemeines Unglück“ nicht als zwangsläufig ungesund aufgefasst. Will man aber bei der Pathologie des Unterbewussten, wie im Fall der Hysterie, von ver- 
schobenen Symptomen ausgehen, ist es notwendig, „das pathogene psychische Material dem Bewußtsein zuzuführen [..., um] so die durch die Bildung von Ersatzsymptomen hervorgerufenen Leiden zu beseitigen“ (Freud 1910, S. 40). Ein zweites Fundament psychischer Gesundheit (neben der grundlegend bedeutenden biologischen und kognitiven Stabilität) ist also eine Form von Einsicht: Selbsterfahrung und Selbsterkenntnis der Person. Nun aber zu einem sozialen Modell.

\subsection{Ein sozialkonstruktivistisches Modell}

Nach Rom Harré (1927-2019) ist das zentrale Thema des Sozialkonstruktivismus die Annahme, dass die meisten psychischen Phänomene sozial konstruiert sind und vorrangig im sozialen Umgang mehrerer Personen miteinander existieren (Harré 1993, S. 95). An anderer Stelle äußert Harré, ein grundlegender Gedanke des Sozialkonstruktivismus sei, dass alle psychischen Phänomene und die Subjekte, in denen sie realisiert werden, diskursiv erzeugt würden (Harré 1992, S. 154, Hervorhebung im Original). Dieser psychologische Ansatz hat großen Einfluss ausgeübt und unter anderem gezeigt, dass das Verhalten einer Person, die mit der Diagnose ,Demenz' lebt, möglicherweise eher durch ihre psychosoziale Umwelt denn durch Hirnpathologie bedingt ist (Sabat 2001).

Es gibt Kritikpunkte, die man dem sozialkonstruktivistischen Modell vorhalten kann (Thornton 2006). Wenn es eine kausale Erklärung für geistige Phänomene liefern soll, scheint es ihm an Weite $\mathrm{zu}$ fehlen, denn es berücksichtigt nicht die biologischen und neurokognitiven Gegebenheiten, d.h. die Mechanismen, ohne welche soziales oder diskursives Geschehen gar nicht erst möglich wäre. Als konstitutiv orientierter Zugang dagegen verleiht es dem Modell des Geistes einiges an Weite. Und dennoch krankt es daran, dass es keine guten Gründe für die Normativität intentionaler Geisteszustände vorbringen kann.

Intentionale Geistesregungen, z.B. etwas meinen, etwas verstehen, sich an etwas erinnern, beziehen sich immer auf ein etwas: wenn ich $\mathrm{X}$ meine, sage ich etwas über X; Erinnerung ist immer Erinnerung an etwas. Intentionale Geistesregungen tragen bereits eine gewisse Normativität in sich, die ihrerseits Teil dieser Zustände ist. Diese geistigen Regungen legen die Welt auf etwas Bestimmtes, und eben nichts anderes, fest. Wer beispielsweise weiß, wie man +2 rechnet, der wird nach der Aufforderung, 2046+2 zu rechnen, notwendig 2048 sagen. Das gehört wesentlich dazu, wenn wir behaupten wollen, die betreffende Person könne +2 rechnen. Die Frage für den Sozialkonstruktivismus lautet: Woher kommt diese Normativität? Ein Sozialkonstruktivist antwortet darauf, es handele sich um eine soziale Konstruktion, ohne dabei jedoch dem Umstand gerecht zu werden, dass 
gerade Normativität ja bereits Voraussetzung dafür ist, dass Bedeutung überhaupt entstehen kann. Ein Philosoph wird vielleicht sagen, es handele sich hier um eine transzendente Voraussetzung, nicht um einen Gegenstand sozialer Vereinbarung, die einer entsprechenden Normativität vorausgegangen wäre (für eine ausführlichere Diskussion siehe Hughes 2011, S. 81-116).

Im Rahmen des Gesundheitsbegriffes in der Psychiatrie führt uns der Sozialkonstruktivismus zu der Erkenntnis, dass psychische Gesundheit zu einem nicht unerheblichen Teil eine Funktion des sozialen Umfeldes ist. Tom Kitwood (1937-1998), der große Befürworter der personenzentrierten Pflege von Demenzerkrankten, greift eine sozialkonstruktivistische Perspektive auf, wenn er Personsein definiert als eine Stellung oder einen Status, der einem menschlichen Wesen von anderen im Kontext von Beziehungen und sozialem Miteinander zugeschrieben wird (Kitwood 1997, S. 8). Kitwood spricht ferner von einer „bösartigen Sozialpsychologie“ (malignant social psychology), die die Stellung einer Person, die an Demenz leidet, untergraben kann (Kitwood 1997, S. 45-49). Sabat spricht in ähnlicher Weise von „bösartiger Positionierung“ (malignant positioning) der Person (Sabat 2001, S. 124-125), an der auch andere ihren Anteil haben, wenn sie, mitunter aus wohlmeinenden Absichten, Dinge tun, die den Betroffenen nichtsdestoweniger degradieren. Unsere psychische Gesundheit hängt also davon ab, dass wir das richtige psychosoziale Umfeld haben.

\section{Zwischenfazit}

Bislang sind wir davon ausgegangen, dass der Gesundheitsbegriff in der Psychiatrie auch gute genetische Anlagen voraussetzt. Diese liefern jedoch weder ein notwendiges noch ein hinreichendes Kriterium für gute psychische Gesundheit (ein Hoch auf Unterschiede!). Außerdem sollten wir Hirntraumata vermeiden und auch unsere physische Gesundheit nicht vernachlässigen (was dem Herzen guttut, ist auch gut für das Gehirn). Und dennoch kann der Zustand des Gehirns weder notwendig mit der An- noch mit der Abwesenheit geistiger Erkrankung in Verbindung gebracht werden, auch wenn gewisse hirnpathologische Befunde die materielle Grundlage für geistige Krankheiten darstellen. Ebenso sind wir davon ausgegangen, dass „gemeines Unglück“ Gesundheit keinesfalls ausschließt, wiewohl eine gewisse Selbsterkenntnis und Selbsterfahrung der Gesundheit natürlich zuträglich sind. Wir haben gesehen, dass gute geistige Gesundheit durch Bildung und soziale Stimulation unterstützt werden kann. Aber wir sind zu einer noch weiteren Perspektive auf den gesamten Organismus - nicht nur auf das Gehirn - gelangt, die den Kontext der menschlichen Welt miteinbezieht, um die Bedeutung geistiger Vorgänge $\mathrm{zu}$ verstehen, seien sie nun krank oder gesund. 
Doch selbst von ,geistigen Vorgängen` zu sprechen, kann irreführend sein (insofern es nahelegt, dass es sich dabei um innere Vorgänge handelt), wenn wir uns vergegenwärtigen, welch großen Einfluss die (äußere) psychosoziale Umwelt auf die psychische Gesundheit ausübt. Unser Augenmerk gilt nun also der Person in der Welt.

Ehe wir jedoch zu dieser Betrachtung der Person gelangen, sollten wir noch ein Wort darüber verlieren, inwiefern die oben diskutierten Modelle in der Praxis einen Unterschied machen. Ich denke, sie machen einen Unterschied; dennoch bleibt die Frage zu beantworten, wie realistisch man davon ausgehen kann, dass jemand sich tatsächlich auf ein bestimmtes Modell festlegt. Das hängt damit zusammen, dass praktizierende Psychiater wissen, dass sie mit Modellen eklektisch umgehen müssen. Es mag hochspezialisierte Einrichtungen geben, in denen ein Modell dominiert, aber generell ist es eher hilfreich, offen für eine größere Bandbreite an Ansätzen zu sein.

Strikt angewandt kann ein biologisches Modell eine Menge erreichen, doch setzt es generell ein gewisses Verständnis der psychischen Konstitution des Patienten voraus (in einem konstitutiven, nicht rein kausalen Sinne), um sein oder ihr Vertrauen für die Behandlung und das behandelnde Team gewinnen zu können. Was soll eine Behandlung auch ausrichten, wenn sie die prekären Verhältnisse unberücksichtigt lässt, in denen die Person lebt? Auf ähnliche Weise kann das Computer-Modell ein sinnvoller Weg sein, um die kognitiven Defizite einer Person zu erklären - ein Weg auch im therapeutischen Sinne. Dennoch wird die Therapie nicht besonders sinnvoll sein, wenn die biochemischen Auffälligkeiten im Gehirn des Betroffenen, die seine Kognition beeinflussen, dabei ignoriert werden. Ähnliches kann man dem psychotherapeutischen Verständnis und dem sozialkonstruktivistischen Ansatz vorhalten. Sie alle erlauben uns hilfreiche Einsichten: Liegt aber ein chemisches Ungleichgewicht vor, dem man abhelfen und so eine Verbesserung des Zustandes herbeiführen könnte - sollte dann dieser Ansatz nicht versucht werden?

Was wir also brauchen, ist ein Ansatz, der die ganze Person in den Blick nimmt, keine Ebene außer Acht lässt und der Person als Person und nicht als Gegenstand eines Modells begegnet. Gesundheit in der Psychiatrie stellt sich so dar als das gesunde Aufeinandertreffen von Personen als solchen.

\section{Zwei Überlegungen und eine Perspektive}

Ehe wir zum Schluss kommen, möchte ich noch zwei weitere Überlegungen anbringen, um eine angemessene Sicht auf das Personsein entwickeln zu können. Die erste betrifft unseren Begriff des Geistes, die zweite Modelle als solche. 


\subsection{Der Geist}

Ich habe skizziert, inwiefern der Geist, verstanden als etwas Inneres, dennoch nicht von der Außenwelt abgeschnitten ist, sondern in Verbindung mit ihr steht. Eine solche Idee des externen Geistes birgt noch weitere Implikationen: Der externalistische Geistesbegriff besagt, dass wir den Inhalt unseres Geistes, das Geistige, nicht charakterisieren können, ohne dabei auf Weltliches, Nicht-Geistiges zu verweisen - frei formuliert nach McCulloch: „der Geist sitzt halt nicht im Kopf“ (McCulloch 2003, S. 12). Wie an anderer Stelle erläutert, setzt sich ein Gutteil unseres geistigen Lebens im externen Raum fort, in dem unser Geist mit Objekten und mit anderen in Beziehung tritt (Hughes 2006). Die Welt ist konstitutiv für den Geist (Hughes 2011, S. 76-78). Wenn unser geistiges Leben also in seiner Einzigartigkeit durch unseren Sprachgebrauch charakterisiert wird, der die Welt mit umfasst, dann sind wir als menschliche Wesen in die Welt eingebettet, und zwar nicht als eine empirische Datengröße, sondern als metaphysische Realität. ${ }^{3}$

\subsection{Modelle}

Heuristisch haben wir nun vom Gebrauch von Modellen profitieren können; dennoch sollten wir festhalten, dass alle Modelle stets auf die eine oder andere Weise Schwachstellen aufwiesen. Wir hatten gehofft, dass die Begriffe sich in die Modelle einklinken würden, und müssen nun sehen, dass unsere Modelle nicht in der Lage sind, die Realität des Geistes zu erfassen. Der Geist sprengt die Modelle sozusagen und greift auf die Welt über. Die Modelle hätten niemals ausreichen können, und so müssen wir zu einer Welt jenseits von Modellen vordringen (Hughes 2011, S. 185-220). Diese Welt ist die Welt tatsächlicher Leute in der tatsächlichen Welt, die sich nicht auf einfache Begriffe herunterbrechen lässt.

\subsection{Personsein}

So gelangen wir zu einer Sicht von Personen, die physisch und metaphysisch in der Welt situiert sind, und in der wir auch leiblich individuierte, selbstbestimmte

3 Allägliche Erfahrung lehrt uns, dass wir in einem empirischen Sinne Teil der Welt sind: Wir bewegen uns in ihr, wir bestehen selbst aus weltlicher Materie usw. Dem metaphysischen Verständnis geht es darüber hinaus darum, dass auch der Geist, recht verstanden, mit der Welt zu tun hat. Er existiert nicht als ein gespenstisches Wesen in vollkommener Loslösung von der Welt. Vielmehr ist die Welt konzeptuell, und nicht bloß empirisch, Teil des geistigen Lebens. 
Handlungsinstanzen (embodied agents) darstellen (Hughes 2001; 2011, S. 29-54). Diese Auffassung der Person (als situierte, leiblich individuierte, selbstbestimmte Handlungsinstanz) lässt sich nicht herunterbrechen oder umschreiben: Die Vielfalt der Möglichkeiten, wie Personen situiert sein können, lässt sich weder modellhaft noch begrifflich voll erfassen. Trifft dies zu, so ist es unwahrscheinlich, dass ein Gesundheitsbegriff, insofern er sich auf Personen bezieht, aus psychiatrischer Sicht jemals festzumachen sein wird.

\section{Schlussfolgerung}

Es gibt in der Psychiatrie nicht den einen, fixen Gesundheitsbegriff; es handelt sich auch nicht um mehrere Gesundheitsbegriffe, die sich nahtlos in eine übergeordnete Gesundheitstheorie integrieren ließen. Dies liegt nicht etwa daran, dass Psychiatrie vage wäre. Es liegt daran, dass in der Psychiatrie die Komplexität der ganzen Person in den Blick genommen wird. Wie Jaspers (1923) gleich zu Beginn seiner Allgemeinen Psychopathologie sagt: „Im praktischen psychiatrischen Berufe handelt es sich immer um einzelne ganze Menschen“ (Jaspers 1923, S. 1, Hervorhebung im Original). Vielmehr muss Gesundheit also jeweils neu verhandelt werden.

Selbst dort, wo chronische Erkrankungen oder regelmäßige Rückfälle vorliegen, bleibt es sinnvoll, von Gesundheit zu sprechen, zumindest in Form von Rehabilitation oder Genesung bzw. recovery (Anthony 1993). Recovery muss dabei nicht gleichbedeutend mit ,Heilung' sein, sondern geht eher - etwa im Fall von Demenz - mit der Vorstellung einher, einen gewissen Lebensstandard zurückzuerlangen oder mit den Umständen gut umgehen zu können (Hill et al. 2021). Die Person selbst ist unausweichlich miteinbezogen, wenn in der Psychiatrie eine Entscheidung zwischen, gesund' und ,nicht gesund‘ zu fällen ist (ob das tatsächlich geschieht, steht auf einem anderen Blatt).

Natürlich kann dies nur funktionieren, sofern die zugrundeliegenden (Wert-) Urteile von allen Seiten geteilt werden. Einmal mehr sind wir bei wertorientiertem Handeln angekommen (Fulford \& van Staden 2013). Wo solche Urteile eben nicht geteilt werden, braucht es gegebenenfalls eine offene Verhandlung. Aber es wird unausweichlich Fälle geben, beispielsweise bei Zwangseinweisungen, wo auch durch Verhandeln kein voller Konsens erreicht wird. Klassischerweise geht es dabei um die Gefahr, die eine Person für sich selbst und für andere darstellt. Wichtig ist hier, dass die Prozesse, die eine Zwangseinweisung ermöglichen, transparent gemacht und unabhängig begutachtet werden. Darüber hinaus sollte die Perspektive der betroffenen Person nicht beiseitegelassen, sondern weiterhin ernstgenommen und respektiert werden. Es handelt sich um die Realität der 
Betroffenen. Dazu passt eine Bemerkung, die Wittgenstein einmal seinem früheren Schüler Maurice O’Connor Drury, einem angehenden Psychiater, gegenüber gemacht hat:

Du darfst nie aufhören, dich über geistige Krankheit zu wundern. Sollte ich psychisch erkranken, dann wäre meine größte Angst die, dass du dem gesunden Menschenverstand folgen könntest, dass du also einfach für gegeben annimmst, ich sei wahnsinnig (Drury 1981, S. 166).

\section{Literatur}

Almeida-Meza, Pamela/Steptoe, Andrew/Cadar, Dorina (2020): „Markers of Cognitive Reserve and Dementia Incidence in the English Longitudinal Study of Ageing“. In: British Journal of Psychiatry, S. 1-9.

Anthony, William Arnold (1993): „Recovery from Mental Illness: The Guiding Vision of the Mental Health Service System in the 1990s“. In: Psychosocial Rehabilitation Journal 16(4), S. 11-23.

Cowen, P. J./Browning, M. (2015): „What Has Serotonin to Do with depression?“. In: World Psychiatry 14(2), S. 158-160.

Drury, Maurice O’Connor (1981): „Conversations with Wittgenstein“. In: Rush Rhees (Hrsg.): Ludwig Wittgenstein - Personal Recollections. Oxford: Blackwell, S. 112-189.

Fodor, Jerry A. (1976): The Language of Thought. Hassocks, Sussex: The Harvester Press. Freud, Sigmund (1910): Über Psychoanalyse. Fünf Vorlesungen gehalten zur 20jährigen Gründungsfeier der Clark University in Worcester Mass., September 1909. Leipzig \& Wien: Deuticke.

Freud, Sigmund/Breuer, Josef (1895): Studien über Hysterie. Leipzig \& Wien: Deuticke.

Fuchs, Thomas (2010): Das Gehirn - ein Beziehungsorgan. Eine phänomenologisch-ökologische Konzeption. Stuttgart, Berlin \& Köln: Kohlhammer.

Fulford, K. W. M. (Bill)/Peile, Ed P./Carroll, Heidi (2012): Essential Values-based Practice: Clinical Stories Linking Science with People. Cambridge: Cambridge University Press.

Fulford, K. W. M. (Bill)/van Staden, C. W. (Werdie) (2013): „Values-based Practice: Topsy-turvy Take-home Messages from Ordinary Language Philosophy (and a Few Next Steps)“. In: K. W. M. (Bill) Fulford/Martin Davies/Richard G. T. Gipps/George Graham/John Z. Sadler/Giovanni Stanghellini/Tim Thornton (Hrsg.): Oxford Handbook of Philosophy and Psychiatry. Oxford: Oxford University Press, S. 385-410.

Griffin, James (1986): Well-Being: Its Meaning, Measurement and Moral Importance. Oxford: Clarendon Press.

Harré, Rom (1992): „What Is Real in Psychology: A Plea for Persons“. In: Theory \& Psychology 2(2), S. $153-158$.

Harré, Rom (1993): Social Being. Oxford: Blackwell.

Heinz, Andreas (2014): Der Begriff der psychischen Krankheit. Berlin: Suhrkamp.

Hill, Laura/Roberts, Glenn/Perkins, Rachel (2021): „What Can Person-centred Care in Dementia Learn from the Recovery Movement?“. In: Philippa Lilford/Julian C. Hughes (Hrsg.): 
Clinical Topics in Old Age Psychiatry. Cambridge: Cambridge University Press,

S. $202-212$.

Hughes, Julian C. (2001): „Views of the Person with Dementia“. In: J Med Ethics 27(2),

S. $86-91$.

Hughes, Julian C. (2006): „Beyond Hypercognitivism: A Philsophical Basis for Good Quality

Palliatice Care in Dementia“. In: Les Cahiers de la Fondation Médéric Alzheimer 2,

S. $17-23$.

Hughes, Julian C. (2011): Thinking Through Dementia. Oxford: Oxford University Press.

Hughes, Julian C. (2016): „Dementia and the Nature of Mind“. In: Geoffrey Scarre (Hrsg.): The

Palgrave Handbook of the Philosophy of Aging. London: Palgrave Macmillan, S. 283-303.

Hughes, Julian C./Williamson, Toby (2019): The Dementia Manifesto: Putting Values-based

Practice to Work. Cambridge: Cambridge University Press.

Jaspers, Karl (1923): Allgemeine Psychopathologie für Studierende, Ärzte und Psychologen

(3., vermehrte u. verbesserte Aufl.). Berlin \& Heidelberg: Springer.

Kitwood, Tom (1997): Dementia Reconsidered: The Person Comes First. Buckingham: Open University Press.

Livingston, Gill/Sommerlad, Andrew/Orgeta, Vasiliki et al. (2017): „Dementia Prevention, Intervention, and Care“. In: The Lancet 390(10113), S. 2673-2734.

Lowe, E. Jonathan (2000): An Introduction to the Philosophy of Mind. Cambridge: Cambridge University Press.

Matthews, Eric (2005): Mind: Key Concepts in Philosophy. London \& New York: Continuum.

McCulloch, Gregory (2003): The Life of the Mind. An Essay on Phenomenological Externalism. London \& New York: Routledge.

Osimo, Emanuele F./Beck, Katherine/Reis Marques, Tiago et al. (2019): „Synaptic Loss in Schizophrenia: A Meta-analysis and Systematic Review of Synaptic Protein and mRNA Measures“. In: Molecular Psychiatry 24(4), S. 549-561.

Sabat, Steven R. (2001): The Experience of Alzheimer's Disease: Life Through a Tangled Veil. Oxford \& Malden, MA: Blackwell.

Schuch, Felipe B./Vancampfort, Davy/Richards, Justin et al. (2016): „Exercise as a Treatment for Depression: A Meta-analysis Adjusting for Publication Bias“. In: Journal of Psychiatric Research 77, S. 42-51.

Snowdon, David A. (2003): „Healthy Aging and Dementia: Findings from the Nun Study“. In: Annals of Internal Medicine 139(5 Pt. 2), S. 450.

Thornton, Tim (2006): „The Discursive Turn, Social Constructionism, and Dementia“. In: Julian C. Hughes/Stephen J. Louw/Steven R. Sabat (Hrsg.): Dementia: Mind, Meaning, and the Person. Oxford: Oxford University Press, S. 123-141.

Tyrer, Peter/Steinberg, Derek (2005): Models for Mental Disorder: Conceptual Models in Psychiatry (4. Aufl.). Chichester: John Wiley \& Sons. 